\title{
The chondrocoracoideus muscle: $A$ rare anatomical variant of the pectoral area
}

\author{
Dionysios Venieratos ${ }^{1}$, Alexandros Samolis ${ }^{1}$, Maria Piagkou ${ }^{1}$, Stergios Douvetzemis ${ }^{1}$, \\ Alexandrina Kourotzoglou ${ }^{1}$, Kontantinos Natsis ${ }^{2}$
}

${ }^{1}$ Department of Anatomy, School of Medicine, Faculty of Health Sciences, National and Kapodistrian University of Athens, Greece, ${ }^{2}$ Department of Anatomy and Surgical Anatomy, School of Medicine Faculty of Health Sciences, Aristotle University of Thessaloniki, Greece

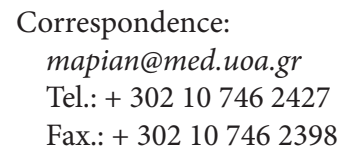

Received: 16 April 2017 Accepted: 12 June 2017

Key words: Pectoralis major - Variation Accessory muscle $\cdot$ Chondrocoracoideus • Thoracic wall muscles.

\begin{abstract}
Objective. The study adds important information regarding the descriptive anatomy of a very rarely reported unilateral chondrocoracoideus muscle (of Wood). Additionally it highlights the concomitant muscular and neural alterations. Case report. The current case presents the occurrence of a chondrocoracoideus muscle situated left-sided, as an extension of the abdominal portion of the pectoralis major muscle (PM). The chondrocoracoideus coexisted with a contralateral atypical PM, partially blended with the clavicular fibers of the deltoid muscle. There was an accessory head of the biceps brachii while the palmaris longus was absent on the right side of a 78-year-old Greek male cadaver. Conclusion. The above mentioned muscular abnormalities are shown as disturbances of embryological pectoral muscle development, and their documentation is essential in order to increase awareness among clinicians of their potential impact on the diagnosis and treatment of several pathologies.
\end{abstract}

\section{Introduction}

The pectoralis major and minor muscles (PM and Pm) are liable to many slight transmutations and are associated with other muscles of the pectoral, axillary and arm region under variable forms. The $\mathrm{PM}$, the largest and most superficial pectoral muscle, typically originates from the medial half of the clavicle, the anterior surface of the sternum, the $2^{\text {nd }}$ to $6^{\text {th }}$ costal cartilages and the external oblique muscle (EOM) aponeurosis, and inserts into the lateral lip of the intertubercular humeral sulcus (1). Atypical, supernumerary or accessory muscles originating from the anterior thoracic wall (sternalis, chondro or costoepitrochlearis, chondro or costohumeralis, pectoralis quartus and axillary arch) join various parts of the pectoral muscles with the medial intermuscular septum, the axillary or brachial fascia, the greater or lesser humeral tubercle, the medial humeral epichondyle, the coracoid process $(\mathrm{CP})$, the shoulder joint capsule and the biceps brachii muscle (BB) $(2,3)$, via epigastric, muscular or tendinous slips (4-6). Rarely, a distinct portion of the inferior fibers of the PM forms a muscular slip and inserts into the $\mathrm{CP}$, superficial to the coracobrachialis muscle (CB), medial to the short head of the $\mathrm{BB}$ and external to the $\mathrm{Pm}$. This muscle is known as the chondroor costocoracoideus muscle (of Wood) (6). 
Pectoral muscle aberrations are usually asymptomatic and are discovered incidentally during surgery or dissection. Their ectopic insertions may provoke neurovascular entrapment, functionally limited abduction of the humerus, and cosmetic deformities $(7,8)$. They also implicate breast and axilla reconstruction. When they produce symptoms, they pose a surgical problem, as it is difficult to differentiate them from soft-tissue tumors (9).

The current report presents the occurrence of a very rarely reported unilateral muscular variant, the chondrocoracoideus muscle, situated on the left side, as an extension of the abdominal portion of the PM. Contralaterally, the muscle coexisted with an atypical PM, an accessory head of the $\mathrm{BB}$, and with an absent palmaris longus. Concomitant neural alterations were also recorded. The detected muscular abnormalities are mentioned as disturbances of the pectoral muscles' development, and their documentation is essential in order to increase awareness among clinicians of their potential impact in the diagnosis and treatment of several pathological entities.

\section{Case report}

During dissection of the thoracic wall, axilla and arm of a 78-year-old Greek male cadaver (death from cardiac arrest), an accessory left-sided chondrocoracoideus muscle (of Wood) was detected, as an extension of the abdominal portion of the PM. On the ipsilateral side, the chondrocoracoideus coexisted with a $\mathrm{CB}$ of one head, arising from the medial border of the tendon of the short head of the BB. The musculocutaneous nerve did not pierce the $\mathrm{CB}$, but it coursed medial to it (Figure 1C). On the contralateral side, an atypical PM coexisted with an accessory head of the $\mathrm{BB}$, while the palmaris longus was absent. On both sides, the PM was divided into a clavicular and a sternocostal portion, with a prominent cleft between them (Figure 1A, and 1D). Both portions inserted via a superficial and a deep flat tendon on the crest of the greater humeral tubercle, the lateral lip of the intertubercular groove, to the point of deltoid muscle insertion. On the right side, the clavicular portion of the PM partially blended with the clavicular fibers of the deltoid muscle. No cephalic vein was found (Figure 1D). The abdominal portion of the right PM was fused to the sternocostal portion, while on the left side the abdominal portion of the PM was represented by the accessory chondrocoracoideus, emerging as three slips from the $6^{\text {th }}-8^{\text {th }}$ ribs and the EOM aponeurosis (Figure 1A, and 1B). At the level of the $5^{\text {th }}$ and $6^{\text {th }}$ ribs, the accessory muscle was fused to the sternocostal portion of the $\mathrm{PM}$ and inserted into the $\mathrm{CP}$ after its fusion with the tendon of the short head of the BB. The chondrocoracoideus was innervated by the medial pectoral nerve, and supplied by the lateral thoracic artery. The short head of the right $\mathrm{BB}$ originated together with the $\mathrm{CB}$ from the tip of the $\mathrm{CP}$, the long head from the supraglenoid tubercle and the posterior part of the glenoid labrum, and the accessory head from the articular humeral capsule (Figure 1E). The accessory head of the BB joined the long head. On the left side, a communication between the musculocutaneous and the median nerve distal to the $\mathrm{CB}$, and the common trunk of the medial brachial and the medial antebrachial cutaneous nerves, arising from the medial cord of the brachial plexus, were observed. 


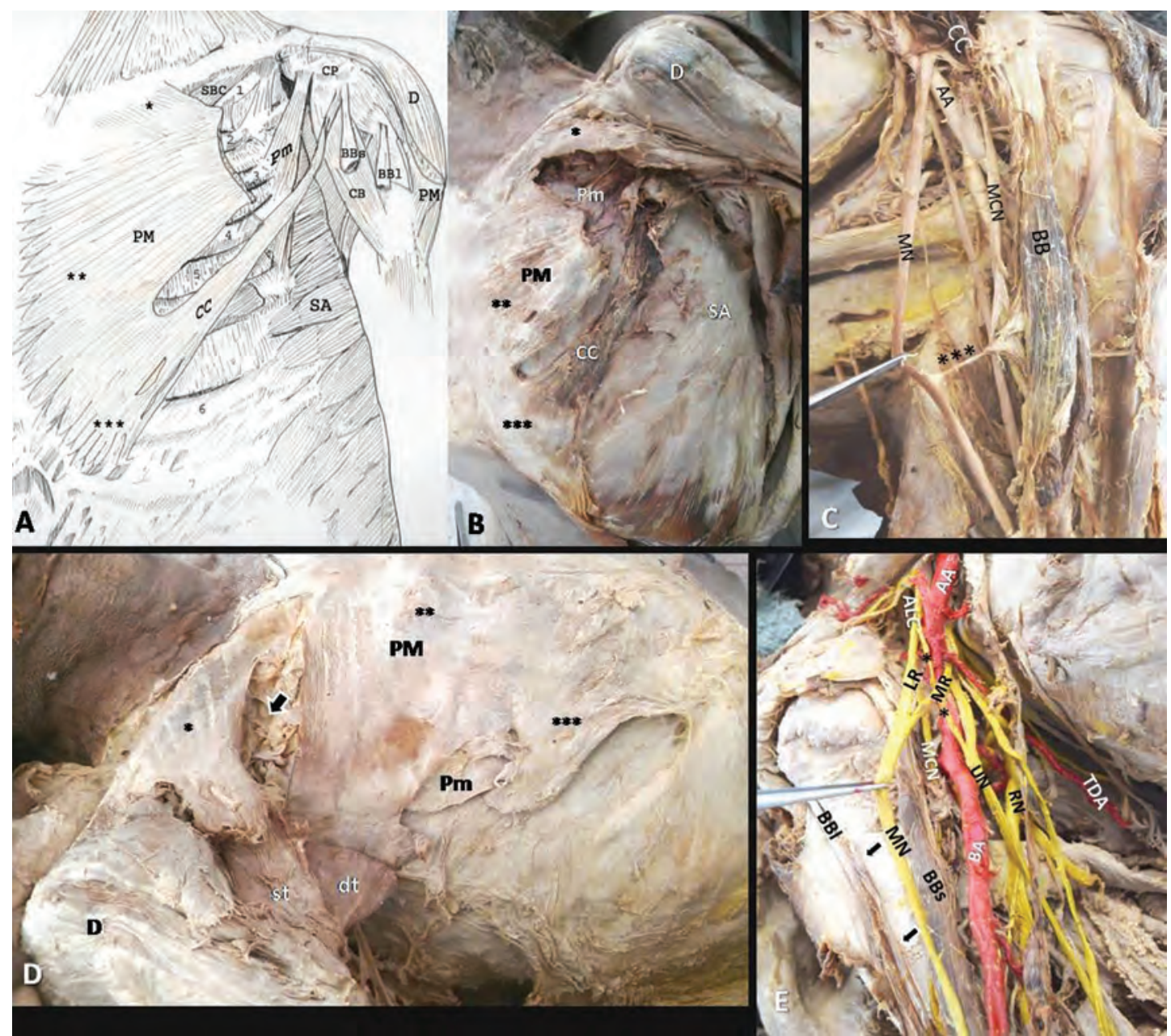

Figure 1 A. Schema and B. Photograph of the left axillopectoral area. The clavicular $\left(^{*}\right)$ and sternocostal portion $\left(^{* *}\right)$ of the pectoralis major (PM). The abdominal portion $\left(^{* *}\right)$ is an accessory pectoral muscle named the chondrocoracoideus muscle (CC) emerging by three slips from the $6^{\text {th }}-8^{\text {th }}$ ribs and the external oblique muscle aponeurosis, and inserted onto the coracoid process (CP) after its fusion with the tendon of the short head of the biceps brachii (BBs). C. Anastomosis $\left({ }^{* *}\right)$ between the musculocutaneous (MCN) and median (MN) nerves distal to the coracobrachialis (CB). The MCN coursed medially to the CB. D. The right PM is divided into a clavicular $\left({ }^{*}\right)$, a sternocostal $\left(^{* *}\right)$ and an abdominal $\left({ }^{* *}\right)$ portion. Muscular fibers of the clavicular portion originated from the medial half of the clavicle and were separated from the sternocostal portion by a large interval (arrow). Both sternocostal and clavicular portions inserted via a superficial (st) and a flat tendon (dt) on the crest of the greater humeral tubercle. The abdominal portion was fused to the sternocostal portion. E. Anastomosis $\left.{ }^{* *}\right)$ between the anterior lateral cord (ALC) of the brachial plexus and the ulnar nerve (UN). BBI-long head of the biceps brachii, D-deltoid, Pm-pectoralis minor, SA-serratus anterior, SBC-subclavius muscle, RN-radial nerve, LR-lateral root, MR-medial root, AA-axillary artery, BA- brachial artery and black arrow- accessory head of the biceps brachii, TDA-thoracodorsal artery, BB-biceps brachii, AA- axillary artery.

\section{Discussion}

Pectoral muscles present a wide variability in type and number of attachments and supernumerary slips $(1,9)$, and may be accompanied by neural variations (10). They also fuse with other muscles of the adjacent (axillary shoulder and arm) area. The presence of aberrant muscular slips may affect the size and origin of the pectoral muscles (7). PM and Pm congenital malformations have an incidence ranging from $0.009 \%$ to 
$0.061 \%$ (11). Developmentally, the pectoral muscles derives from a primitive muscle $\left(5^{\text {th }}\right.$ to $7^{\text {th }}$ cervical myotomes) during the $5^{\text {th }}$ fetal week. A combination of migration, fusion and apoptosis of myogenic cell precursors gives the muscles their final shape. Concerning the abdominal portion of the $\mathrm{PM}$, it is considered to be a derivate of the "panniculus carnosus" in mammals (12). In higher primates and humans, the "panniculus carnosus" regresses during evolution in favor of wide upper limb mobility, and remnants are found as: i) extra muscular slips from the EOM aponeurosis attached to the serratus anterior fascia, ii) separate bundles arising below the PM and inserted at the axillary fascia, the pectoral humeral ridge, the fascia between the CB and Pm, or onto the $\mathrm{CP}$, iii) slips from the latissimus dorsi (LD) border extended to the axillary or brachialis fascia, the pectoral region, the humerus, or the CP $(1,13)$. The chondrocoracoideus may also be considered as an atavistic anomaly, demonstrating the extent of PM insertion in ancestral forms. Concerning PM variants, the fusion of the PM with the deltoid muscle is the simplest deviation, while other unions with the rectus abdominis muscle, the $\mathrm{BB}$, the $\mathrm{LD}(1)$, the contralateral PM (5) and the sternocleidomastoid muscle (as the rectus thoracis bifurcalis) (14) are more uncommon. A deltopectoral complex is created when the lateral border of the PM clavicular portion is totally fused with the medial border of the deltoid (15). In such cases, the cephalic vein is either aborted, or reaches the axillary vein. More rarely, the PM may be unilaterally or bilaterally partially (sternal segment or clavicular head) or totally absent (5). Alternatively, the PM may be divided into two slips, with the external slip coursing behind the long head of the $\mathrm{BB}$ and inserted into the lateral lip of the intertubercular sulcus, or may be inserted into the CP and CB fascia, or into the glenohumeral joint capsule via an accessory slip, the chondrocoracoideus muscle, so called by Wood and also described by Macalister and Perrin, dating back to the $19^{\text {th }}$ century. The chondrocoracoideus is one of the rarest remnants of the panniculus (of skin-associated musculature), arising only on the left side from the $5^{\text {th }}$ and $6^{\text {th }}$ ribs and the EOM aponeurosis, coursing between the PM and $\mathrm{LD}$, and separating the lateral from the posterior cord of the brachial plexus $(1,5,6)$. In our case, the left chondrocoracoideus originated in three slips from the $6^{\text {th }}-8^{\text {th }}$ ribs and the EOM aponeurosis, and inserted with the Pm into the CP. Bannur et al. (9) reported a right-sided accessory muscle that originated from the $5^{\text {th }}$ and $6^{\text {th }}$ ribs at the costochondral junction, fused laterally with the Pm and inserted into the CP. It is unknown if the chondrocoracoideus is a developmental variant of the PM or the Pm, since Tountas and Bergman (1) described the aberrant muscle in Pm variations, probably following its insertion into the $\mathrm{CP}$, while Testut (5) and Le Double (4) reported the muscle as a $\mathrm{PM}$ variation, considering its origin from the lower ribs and the EOM aponeurosis. In our opinion, the chondrocoracoideus is an extension of the abdominal PM portion. Other variable insertions of the PM include the articular humeral capsule, the medial humeral epicondyle (chondro or costohumeralis muscle), the internal brachial ligament or the brachial fascia (chondrofascialis) (5), the medial intermuscular septum, and the epitrochlea (chondro or costo and sterno-costo-epitrochlearis) via muscular, tendinous or epigastric slips (11).

The Pm may present variations at its costal origins (appearing as pectoralis minimus, extending from the $1^{\text {st }} \mathrm{rib}$ to the $\mathrm{CP}$, as sternochondrocoracoideus, extending from the $3^{\text {rd }}$ cartilage, the border of the sternum to the $\mathrm{CP}$, and as pectoralis intermedius, arising from the $3^{\text {rd }}$ and $4^{\text {th }}$ ribs, between the $\mathrm{PM}$ and the $\mathrm{Pm}$ ) and insertions (scapular, capsular, humeral and clavicular) $(1,5)$. The 
Pm may blend with the PM, may be divided into two portions, or may be unilaterally or bilaterally absent (1).

PM anomalies are of paramount importance for interventional clinicians and plastic surgeons, in cases where the muscle is used as a graft during turnover flap removal, for coverage of life-threatening sternal wound infections after sternotomy, in order to avoid sternal osteomyelitis, mediastinitis and systemic sepsis development (16), as well as sternoclavicular joint septic arthritis (17). PM total or segmental flap removal may be used in local mediastinal wounds (18), and in head and neck surgery (19) for pharyngoesophageal and oromandibular reconstruction (20). PM deficits frequently coexist with Pm absence, as part of Poland's syndrome. These deficits make breast reconstruction and final symmetry extremely challenging (21). PM absence also precludes breast implant insertion, and may increase the risk of the direct spread of breast cancer into the thoracic cavity, worsening the disease prognosis $(22,23)$.

The chondrocoracoideus, similarly to the axillary arch, may have the potential to compress the axillary artery and the brachial plexus branches, thus mimicking clinical manifestations of the thoracic outlet syndrome (11). Pm anomalous insertions may cause (24) neurovascular obstruction due to the muscle's close proximity to axillary vessels and brachial plexus. Other clinical consequences include: impingement syndrome, superior labrum anterior to posterior lesions, shoulder pain, snapping sensation and clicking. An ectopic Pm tendon, inserting onto the supraspinatus muscle, may cause pain, stiffness and diminished range in external rotation (25). A Pm flap may be used in facial palsy, in breast, axilla $(21,22)$ and glenohumeral joint reconstruction (26). Knowledge of pectoral muscle variants allows surgeons to be better prepared when performing axillary lymphadenectomy. In such cases, the procedure is carried out with extreme difficulty due to the limited surgical field, and if surgeons navigate one level above the axillary vein, the neurovascular axillary bundle may be injured (11).

\section{Conclusion}

Accessory thoracic wall muscles present a wide variability, and may coexist with other muscular variations in the axillary, shoulder and arm area, within a disturbance in pectoral muscle development. Neural variations may also coexist. The described left chondrocoracoideus muscle is a very rare muscular variant with an uncertain clinical background. Documentation of such rare variants, with their embryological origin, is essential in order to increase awareness among clinicians of their potential impact in diagnosis of several pathologic entities, and of possible complications during surgical manipulation of the affected area. Knowledge of the variable anatomy of the axilla is necessary for surgeons who perform axillary lymphadenectomy and reconstruct the breast area, in order to achieve symmetry and a favorable cosmetic outcome. Cases of neurovascular disorders, limited abduction of the humerus and cosmetic deformities can be explained if these muscular aberrations are kept in mind.

\footnotetext{
What is already known on this topic

The pectoralis major and minor (PM and Pm) are associated with variable forms of muscles of the pectoral, axillary and arm region. Atypical, supernumerary or accessory muscles (sternalis, chondro or costoepitrochlearis, chondro or costohumeralis and axillary arch) are described in the literature. A rare muscle known as the chondro- or costocoracoid muscle was described by Wood, as a muscular formation originating from the inferior fibers of the PM and inserting into the coracoid process, superficial to the coracobrachialis muscle, medial to the short head of biceps brachii and external to the Pm.
}

\section{What this study adds}

The study adds important information regarding the descriptive anatomy of a very rarely reported unilateral chondrocoracoideus muscle (of Wood) situated on the left side, as an 
extension of the abdominal portion of the PM. Additionally, it highlights the concomitant muscular (atypical contralateral $P M$, accessory head of contralateral biceps brachii and palmaris longus absence) and neural alterations. These muscular abnormalities are mentioned as disturbances of pectoral muscle development, and their documentation is essential in order to increase awareness among clinicians of their potential impact in the diagnosis and treatment of several pathologic entities.

Acknowledgements: We thank the male subject for his body donation before death, after giving written informed consent.

Authors' contributions: Conception and design: KN and MP; Cadaver dissection: DV and MP; Acquisition, analysis and interpretation of data: $\mathrm{MP}, \mathrm{AS}$ and $\mathrm{KN}$; Drafting the article: MP, AS, SD, AK and KN; Revising it critically for important intellectual content: DV and $\mathrm{KN}$; Approved final version of the manuscript: DV, AS, MP, SD, AK and KN.

Conflict of interests: The authors declare that there are no conflicts of interests.

\section{References}

1. Tountas CP, Bergman RA. Anatomic Variations of the Upper Extremity, New York: Churchill Livingstone; 1983. p. 196-210.

2. Macalister A. Additional observations on muscular anomalies in human anatomy (third series), with a catalogue of the principal muscular variations hitherto published. Trans Roy Irish Acad Sci. $1875 ; 25: 1-134$.

3. Je SS, Park B, Kim J, Yoon SP. Five-headed biceps brachii muscle with a rare origin from the tendon of pectoralis major muscle. Anat Sci Int. 2016; 91(1):110-3.

4. Le Double AF. Treatise on the variations of the muscular system of man and their significance from the point of view of zoological anthropology [in French]. Paris: Schleicher frères; 1897. p. 252-5.

5. Testut L. Muscle abnormalities in man [in French]. Paris: Masson; 1884. p. 34-44.

6. Wood J. Variations in human myology observed during the winter session of 1867-68 at King's College London. Proc Roy Soc Lon. 1867; 16:483-525.

7. Loukas M, Louis RG Jr, Kwiatkowska M. Chondroepitrochlearis muscle, a case report and a suggested revision of the current nomenclature. Surg Radiol Anat. 2005; 27(4):354-6.

8. Samuel VP, Vollala VR. Unusual pectoralis major muscle: the chondroepitrochlearis. Anat Sci Int. 2008; 83(4):277-9.
9. Bannur BM, Mallashetty N, Endigeri P. An accessory muscle of pectoral region. J Clin Diagn Res. 2013; 7(9):1994-5.

10. Loukas M, Louis RG Jr, Fogg QA, Hallner B, Gupta AA. An unusual innervation of pectoralis $\mathrm{mi}-$ nor and major muscles from a branch of the intercostobrachial nerve. Clin Anat. 2006; 19(4):347-9.

11. Natsis K, Vlasis K, Totlis T, Paraskevas G, Noussios G, Skandalakis P, et al. Abnormal muscles that may affect axillary lymphadenectomy: Surgical anatomy. Breast Cancer Res Treat. 2010; 120(1):77-82.

12. Carlson BM. Human embryology and developmental biology. 3rd ed. Philadelphia, PA: Mosby; 2004. p. 224-5.

13. Yazar F, Kirici Y, Ozan H. Accessory insertions of pectoralis major muscle to the brachial fascia: a case report. Kaibogaku Zasshi. 1998; 73(6):637-9.

14. Mehta V, Arora J, Yadav Y, Suri RK, Rath G. Rectus thoracis bifurcalis: a new variant in the anterior chest wall musculature. Rom J Morphol Embryol. 2010; 51(4):799-801.

15. Natsis K, Tsakotos G, Vlasis K, Totlis T, Koebke J. Absence of the deltopectoral groove. ANZ J Surg. 2011; 81(3):204.

16. Lee CH, Hsien JH, Tang YB, Chen HC. Reconstruction for sternal osteomyelitis at the lower third of sternum. J Plast Reconstr Aesthet Surg. 2010; 63(4):633-41.

17. Schmidt-Rohlfing B, Haas V, Vodopianov M, Kuhtin O. Use of a pediculed pectoralis major flap for the treatment of septic arthritis of the sternoclavicular joint [in German]. Oper Orthop Traumatol. 2014; 26(3):288-94.

18. Sano K, Hyakusoku H, Tanuma K. Clinical reappraisal of the segmental pectoralis major turnover flap for coverage of the local mediastinal wound. Scand J Plast Reconstr Surg Hand Surg. 2005; 39(5):290-4.

19. Tripathi M, Parshad S, Karwasra RK, Singh V. Pectoralis major myocutaneous flap in head and neck reconstruction: An experience in 100 consecutive cases. Natl J Maxillofac Surg. 2015; 6(1):37-41.

20. Sharaf B, Xue A, Solari MG, Boa O, Liu J, Hanasono $\mathrm{MM}$, et al. Optimizing outcomes in pharyngoesophageal reconstruction and neck resurfacing: 10-year experience of 294 cases. Plast Reconstr Surg. 2017; 139(1):105-19.

21. Huber KM, Boyd TG, Quillo AR, Wilhelmi BJ. Implications of anomalous pectoralis muscle in reconstructive breast surgery: the oblique pectoralis anterior. Eplasty. 2012;12:e44.

22. Manstein CH, Manstein G, Somers RG, Barwick WJ. Use of pectoralis minor muscle in immediate 
reconstruction of the breast. Plast Reconstr Surg. 1985;76(4):566-9.

23. Shah R, Kang N. Reconstruction in the axilla with a pedicled pectoralis minor muscle flap. Ann $\mathrm{R}$ Coll Surg Engl. 2006;88(4):9-11.

24. Low SC, Tan SC. Ectopic insertion of the pectoralis minor muscle with tendinosis as a cause of shoulder pain and clicking. Clin Radiol. 2010;65(3):254-6.
25. Moineau G, Cikes A, Trojani C, Boileau P. Ectopic insertion of the pectoralis minor: implication in the arthroscopic treatment of shoulder stiffness. Knee Surg Sports Traumatol Arthrosc. 2008; 16(9):869-71.

26. van de Sande MA, Cosker T, McDonnell SM, Gibbons CL, Giele H. Use of the composite pedicled pectoralis minor flap after resection of soft tissue sarcoma in reconstruction of the glenohumeral joint. Case Rep Orthop. 2014:937342. 